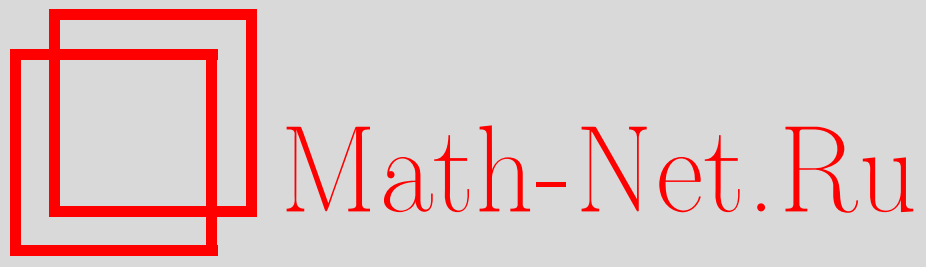

Ю. Б. Орочко, Индексы дефекта симметрического обыкновенного дифференциального оператора с бесконечным числом точек вырождения, Функи. анализ и его прил., 2004, том 38, выпуск 2, 55-64

DOI: https://doi.org/10.4213/faa107

Использование Общероссийского математического портала MathNet.Ru подразумевает, что вы прочитали и согласны с пользовательским соглашением

http://www.mathnet.ru/rus/agreement

Параметры загрузки:

IP : 54.89 .56 .158

26 апреля 2023 г., 09:39:22

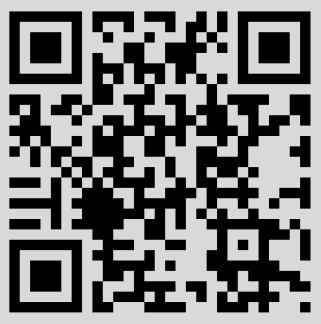




\title{
Индексы дефекта симметрического обыкновенного дифференциального оператора с бесконечным числом точек вырождения*
}

\author{
(C) 2004. Ю. Б. ОРОчКО
}

\section{§1. Введение}

Рассмотрим дифференциальное выражение $s[f]=(-1)^{n}\left(c(x) f^{(n)}\right)^{(n)}, x \in \mathbb{R}$, порядка $2 n \geqslant 2$, коэффициент $c(x)$ которого является действительной функцией, обладающей следующими тремя свойствами: 1) ее множество нулей счетно и не имеет конечных точек накопления; 2) эта функция бесконечно дифференцируема в любой точке $x$, в которой $c(x) \neq 0 ; 3) c^{(n)}(x) \in L_{\mathrm{loc}}^{2}(\mathbb{R})$.

Обозначим через $H$ симметрический минимальный оператор в гильбертовом пространстве $L^{2}(\mathbb{R})$, порожденный дифференциальным выражением $s[f]$, вырождающимся в нулях функции $c(x)$; мы понимаем его как замыкание опеpатора $f \rightarrow s[f]$ с областью определения $C_{0}^{\infty}(\mathbb{R})$. Будем обозначать через Def $A$ значение индекса дефекта действительного симметрического оператора $A$, действующего в гильбертовом пространстве $L^{2}(J), J \subseteq \mathbb{R}$, в верхней (или, что то же самое, нижней) открытой комплексной полуплоскости. Нас будет интересовать значение дефектного числа Def $H$.

В работе [1] показано, что если $c(x)$ - тригонометрический многочлен, удовлетворяющий определенным условиям, то для соответствующего оператора $H$ справедливо равенство Def $H=+\infty$. В настоящей статье получены общие условия на коэффициент $c(x)$ произвольного вида, достаточные для справедливости одной из формул $\operatorname{Def} H=+\infty$ и $\operatorname{Def} H=0$. Особенностью этих условий является то, что в них главную роль играет нетрадиционное понятие двустороннего порядка нуля коэффициента.

Наиболее просто это понятие вводится в случае, когда $c(0)=0$. Будем говорить, что пара чисел $p_{-}>0, p_{+}>0$ задает двусторонний порядок нуля $x=0$ функции $c(x)$, если на некоторых отрезках $\left[-h_{-}, 0\right],\left[0, h_{+}\right]$справедливы представления

$$
c(x)=(-x)^{p_{-}} a_{-}(x), \quad x \in\left[-h_{-}, 0\right], \quad c(x)=x^{p_{+}} a_{+}(x), \quad x \in\left[0, h_{+}\right],
$$

с функциями $a_{-}(x) \in C^{\infty}\left[-h_{-}, 0\right], a_{+}(x) \in C^{\infty}\left[0, h_{+}\right]$, строго положительными или строго отрицательными на соответствующих отрезках. Двусторонний порядок произвольного нуля $x_{0}$ коэффициента $c(x)$ равен $\left(p_{-}, p_{+}\right)$, если функция $c\left(x_{0}+x\right)$ имеет в точке $x=0$ нуль указанного двустороннего порядка. Значения параметров $p_{-}, p_{+}$, задающих соответственно левосторонний и правосторонний порядки нуля $x_{0}$ функции $c(x)$, могут быть любыми положительными числами.

*Работа выполнена при частичной финансовой поддержке РФФИ (грант №02-01-00997). 
Ю. Б. Орочко

В то же время если $c(x) \in C^{\infty}(\mathbb{R})$, то любой нуль $x_{0}$ этой функции двустороннего порядка $\left(p_{-}, p_{+}\right)$является ее нулем в классическом смысле некоторой целой кратности $p \geqslant 1$; при этом справедливо равенство $p_{-}=p_{+}=p$.

Свойство $c^{(n)}(x) \in L_{\text {loc }}^{2}(\mathbb{R})$ коэффициента $c(x)$ дифференциального выражения $s[f]$, порождающего симметрический минимальный оператор $H$, необходимо для корректности используемого нами определения этого оператора. Если любой нуль этого коэффициента имеет некоторый двусторонний порядок, то это свойство имеет место тогда и только тогда, когда значения параметров $p_{-}, p_{+}$, задающие этот порядок, либо больше $n-1 / 2$, либо принимают только целые значения $1,2, \ldots, n-1$ из промежутка $(0, n-1 / 2]$. В данной работе рассматриваются коэффициенты $c(x)$, порядки нулей которых больше $n-1 / 2$. Основным результатом данной статьи является

ТЕорема 1. Если коэффициент $c(x)$ дифференииального выражсения $s[f]$ порядка $2 n \geqslant 2$, порождающего оператор $H$, имеет бесконечную последовательность нулей, двусторонний порядок $\left(p_{-}, p_{+}\right)$каждого из которых удовлетворяет условию $n-1 / 2<p_{-}, p_{+}<2 n-1 / 2$, то Def $H=+\infty$.

Точность присутствующей в условии теоремы 1 оценки сверху на $p_{-}, p_{+}$установлена в нашем втором результате, в котором через $H(J), J \subset \mathbb{R}-$ ограниченный или неограниченный интервал, обозначается симметрический минимальный оператор, порожденный дифференциальным выражением $s[f]$ в $L^{2}(J)$. При этом $L^{2}(J)$ рассматривается как подпространство гильбертова пространства $L^{2}(\mathbb{R})$, состоящее из функций $f(x) \in L^{2}(\mathbb{R})$, равных нулю на множестве $\mathbb{R} \backslash J$, а $H(J)$ - как оператор, действующий в этом подпространстве.

ТЕОРЕмА 2. Пусть нули коэфбициента $c(x)$ дифберенциального выражения $s[f]$ порядка $2 n \geqslant 2$ образуют возрастающую последовательность $x_{r}$, $-\infty<r<+\infty, \lim _{r \rightarrow \pm \infty} x_{r}= \pm \infty$, которая расщепляет числовую ось на счетное множество интервалов вида $J_{r}=\left(x_{r-1}, x_{r}\right)$. Рассмотрим наряду с оператором $H$, порожденным дифберенциальным выражением $s[f]$ в $L^{2}(\mathbb{R})$, бесконечную последовательность операторов $H\left(J_{r}\right),-\infty<r<+\infty$. Если двусторонний порядок $\left(p_{-}, p_{+}\right)$любого нуля коэфбициента $c(x)$ удовлетворяет условию $p_{-}, p_{+} \geqslant 2 n-1 / 2$, то оператор $H$ самосопряжен. Более того, при указанных условиях самосопряжен каждый из операторов $H\left(J_{r}\right),-\infty<r<+\infty$, и справедливо ортогональное разложение $H=\bigoplus_{r=-\infty}^{+\infty} H\left(J_{r}\right)$.

В доказательствах сформулированных теорем используются простые факты теории расширений симметрических дифференциальных операторов и применяются классические асимптотические методы теории обыкновенных линейных дифференциальных уравнений. Трудности, возникающие в этих доказательствах, связаны, главным образом, с большим объемом и громоздкостью присутствующих в них вычислений.

Очевидным следствием теорем 1 и 2 является

Теорема 3. Рассмотрим дифференциалъное выражсение $s[f]$ порядка $2 n \geqslant 2$ c коэбфициентом $c(x) \in C^{\infty}(\mathbb{R})$, нули которого образуют возрастающую последовательность $x_{r},-\infty<r<+\infty, \lim _{r \rightarrow \pm \infty} x_{r}= \pm \infty$. Индексъ дефекта соответствующего оператора $H$ равны нулю, если кратность каждого нуля функиии с $(x)$ (понимаемая в классическом смысле) больше или равна $2 n$. Вместе с тем они равны $+\infty$, если бесконечно много нулей этой функции имеют кратности $p$, удовлетворяющие неравенству $n \leqslant p \leqslant 2 n-1$. 


\section{§2. Доказательство теоремы 2}

Обозначим через $x^{*}$ один из нулей коэффициента $c(x)$ дифференциального выражения $s[f]$. Доказательства теорем 1, 2 получены с помощью своеобразного метода локализации, сводящего изучение индексов дефекта оператора $H$ к такой же задаче для оператора $H(J)$, отвечающего интервалу $J$ одного из следующих трех типов: 1) $J=\left(x^{*}, b\right), c(x) \neq 0$ при $\left.x \in\left(x^{*}, b\right] ; 2\right) J=\left(a, x^{*}\right)$, $c(x) \neq 0$ при $\left.x \in\left[a, x^{*}\right) ; 3\right) J=(a, b), x^{*} \in(a, b), c(x) \neq 0$ при $x^{*} \neq x \in[a, b]$. Так как точку $x=0$ можно сдвинуть в любой нуль $x^{*}$ коэффициента $c(x)$ двустороннего порядка $\left(p_{-}, p_{+}\right)$, то можно считать, что $x^{*}=0, a=-h_{-}<0$, $b=h_{+}>0$ и использовать представление $(1)$ коэффициента $c(x)$ на отрезке $\left[-h_{-}, h_{+}\right]$.

Рассмотрим дифференциальное выражение $s[f]$, коэффициент $c(x)$ которого имеет нуль двустороннего порядка $\left(p_{-}, p_{+}\right)$в точке $x=0$. Тогда можно найти интервал $I_{0}=\left(-h_{-}, h_{+}\right)$, на замыкании которого - отрезке $\left[-h_{-}, h_{+}\right]-$ имеет место представление (1) этого коэффициента. Если расщепить интервал $I_{0}$ точкой $x=0$ на подынтервалы $I_{-}=\left(-h_{-}, 0\right)$ и $I_{+}=\left(0, h_{+}\right)$, то действие дифференциального выражения $s[f]$ на функции $f(x)$, принадлежащие классам $C_{0}^{\infty}\left(I_{-}\right)$и $C_{0}^{\infty}\left(I_{+}\right)$, можно записать соответственно в форме $s[f]=s_{-}[f]$ и $s[f]=s_{+}[f]$, причем вспомогательные дифференциальные выражения, присутствующие в правых частях этих формул, имеют вид

$$
\begin{aligned}
& s_{-}[f]=(-1)^{n}\left((-x)^{p_{-}} a_{-}(x) f^{(n)}\right)^{(n)}, \quad x \in I_{-}, \\
& s_{+}[f]=(-1)^{n}\left(x^{p_{+}} a_{+}(x) f^{(n)}\right)^{(n)}, \quad x \in I_{+} ;
\end{aligned}
$$

первое из них вырождается в правом конце $x=0$ интервала $I_{-}$, а второе - в левом конце $x=0$ интервала $I_{+}$. Наряду с оператором $H\left(I_{0}\right)$, порожденным рассматриваемым дифференциальным выражением $s[f]$ в $L^{2}\left(I_{0}\right)$, введем минимальный оператор $H\left(I_{-}\right)$в $L^{2}\left(I_{-}\right)$, порожденный дифференциальным выражением $s_{-}[f]$, а также минимальный оператор $H\left(I_{+}\right)$в $L^{2}\left(I_{+}\right)$, порожденный дифференциальным выражением $s_{+}[f]$. Для получения свойств оператора $H\left(I_{0}\right)$, используемых в доказательствах теорем 1,2 , сначала исследуются индексы дефекта указанных вспомогательных операторов. Такое исследование достаточно проводить только для оператора $H\left(I_{+}\right)$в силу следующего замечания.

ЗАмЕчАНиЕ 1 . При замене аргумента $x \rightarrow-x$ оператор $H\left(I_{-}\right)$переходит в оператор $H\left(I_{+}\right)$, порожденный дифференциальным выражением $s_{+}[f]$ с параметром $p_{+}=p_{-}$и функцией $a_{+}(x)=a_{-}(-x)$. Поэтому свойства операторов $H\left(I_{+}\right), H\left(I_{-}\right)$, используемые в доказательствах теорем 1,2 , аналогичны. Это обстоятельство позволяет ограничиться доказательством их только для первого оператора.

При каждом значении параметра $p_{+}>0$ дефектное подпространство $\mathfrak{N}_{+} \subset$ $L^{2}\left(I_{+}\right)$симметрического оператора $H\left(I_{+}\right)$, отвечающее фиксированному числу $\bar{z}, \operatorname{Im} z \neq 0$, есть линеал, состоящий из решений дифференциального уравнения

$$
s_{+}[y](x)=(-1)^{n}\left(x^{p_{+}} a_{+}(x) y^{(n)}\right)^{(n)}(x)=z y(x), \quad x \in I_{+},
$$

принадлежащих $L^{2}\left(I_{+}\right)$. Так как $a_{+}(x) \in C^{\infty}\left[0, h_{+}\right]$, то эти решения бесконечно дифференцируемы на промежутке $\left(0, h_{+}\right]$. Из-за вырождения дифференциального уравнения (3) в точке $x=0$ среди функций $y_{k}(x), 1 \leqslant k \leqslant 2 n$, составля- 
ющих его фундаментальное семейство решений, могут быть как функции, входящие в пространство $L^{2}\left(I_{+}\right)$, так и функции, не входящие в него, причем эти свойства зависят от поведения функции $y_{k}(x)$ при $x \rightarrow+0$. Совокупность функций $y_{k}(x)$, принадлежащих $L^{2}\left(I_{+}\right)$, образует базис дефектного подпространства $\mathfrak{N}_{+}$оператора $H\left(I_{+}\right)$, а дефектное число Def $H\left(I_{+}\right)$равно числу элементов этого базиса. Таким образом, нахождение значения последнего числа сводится к исследованию поведения решений дифференциального уравнения (3) при $x \rightarrow+0$. Такое исследование приводит к результатам, сформулированным ниже в леммax 1 и 2.

В лемме 1 используются две серии действительных чисел

$$
\begin{aligned}
\lambda_{k} & =-(k-1), & & 1 \leqslant k \leqslant n, \\
\lambda_{k}\left(p_{+}\right) & =p_{+}-(k-1), & & n+1 \leqslant k \leqslant 2 n ;
\end{aligned}
$$

они возникают как собственные значения некоторой числовой матрицы порядка $2 n$, зависящей от параметра $p_{+}$и связанной с дифференциальным уравнением (3). Существенным свойством этих серий является то, что их пересечение непусто при $p_{+}=1, \ldots, 2 n-1$ и является пустым множеством для всех остальных значений $p_{+}>0$. Будем считать, что кратность каждого из чисел (4) равна 1 , если это число не принадлежит пересечению указанных серий, и равна 2 , если оно принадлежит их пересечению.

ЛЕмма 1. 1) При нецелых значениях параметра $p_{+} \in(0,2 n)$ (в этом случае каждое из чисел (4) однократно) дифференииальное уравнение (3) имеет фундаментальное семейство решений $y_{k}(x), 1 \leqslant k \leqslant 2 n$, с асимптотикой при $x \rightarrow+0$ вида

$$
y_{k}(x)= \begin{cases}x^{-\lambda_{k}}(1+o(1))=x^{k-1}(1+o(1)), & 1 \leqslant k \leqslant n, \\ x^{-\lambda_{k}(p)}(1+o(1))=x^{k-1-p_{+}}(1+o(1)), & n+1 \leqslant k \leqslant 2 n .\end{cases}
$$

2) В случае значений параметра $p_{+}=1, \ldots, 2 n-1$ у дифференииалъного уравнения (3) есть фундаментальное семейство решений $y_{k}(x), 1 \leqslant k \leqslant 2 n$, обладающее следующими свойствами. Во-первых, для каждой из функиий $y_{k}(x)$, $1 \leqslant k \leqslant n$, и тех функиий из набора $y_{k}(x), n+1 \leqslant k \leqslant 2 n$, которые отвечают числам $\lambda_{k}\left(p_{+}\right)$кратности 1 , сохраняется асимптотика при $x \rightarrow+0$, приведенная в формуле (5). Во-вторых, асимптотики при $x \rightarrow+0$ решений $y_{k}(x)$, отвечаюших двукратным числам $\lambda_{k}\left(p_{+}\right)$второй из серий $(4)$, получаются путем замень в соответствующих асимптотиках (5) множителей $x^{-\lambda_{k}\left(p_{+}\right)}$ выражениями $x^{-\lambda_{k}\left(p_{+}\right)} \ln x$.

3) Все перечисленнье асимптотики можно дифференцировать $2 n-1$ раз.

При изучении асимптотики в нуле решений дифференциального уравнения (3) случаи, когда $p_{+}=2 n$ или $p_{+}>2 n$, качественно отличны от случаев, рассмотренных в лемме 1 , а также друг от друга.

ЛЕмма 2. Если $p_{+}>2 n$, дифференциальное уравнение (3) имеет фундаментальное семейство решений $y_{k}(x), 1 \leqslant k \leqslant 2 n$, такое, что при $1 \leqslant k \leqslant n$ для функиий $y_{k}(x)$ u их производных до порядка $2 n-1$ включительно справедлива оценка $O\left(x^{l}\right), x \rightarrow+0$, а для $n+1 \leqslant k \leqslant 2 n$ функиии $\left|y_{k}(x)\right|$ возрастают при $x \rightarrow+0$ бистрее, чем $x^{-l}$, со сколь угодно больиим показателем степени $l$ в обоих случаях. При $p_{+}=2 n$ и достаточно малых значениях числа $z$, 
$\operatorname{Im} z \neq 0$, у дифференциального уравнения (3) есть фундаменталъное семейство решений с $2 n-1$ раз дифферениируемой асимптотикой при $x \rightarrow+0$ вида $y_{k}(x)=x^{n-k+\delta_{k}(z)}(1+o(1)), 1 \leqslant k \leqslant 2 n$, в которой $\delta_{k}(z)-$ числа, обладающие свойствами $\left|\delta_{k}(z)\right|<1 / 4, \operatorname{Im} \delta_{k}(z) \neq 0$.

Для доказательства утверждений, приведенных в леммах 1, 2, мы преобразуем (с помощью специального приема, изложенного в $[2$, гл. $4, \S 12])$ дифференциальное уравнение (3) в эквивалентную систему дифференциальных уравнений, зависящую от параметра $p_{+}$, в которой затем делаем замену аргумента $x=e^{-t}$. В результате этих преобразований вычисление асимптотики при $x \rightarrow+0$ решений $y(x)$ дифференциального уравнения (3) и их производных до порядка $2 n-1$ включительно сводится к нахождению асимптотики при $t \rightarrow+\infty$ решений $\mathbf{v}(t)=\left\{v_{j}(t)\right\}_{j=1}^{2 n}$ некоторой системы дифференциальных уравнений одного из трех хорошо изученных (в асимптотической теории линейных дифференциальных систем) типов, зависящих от значений параметра $p_{+}$. После этого для получения результатов, изложенных в леммах 1,2 , остается применить к указанной системе дифференциальных уравнений классические теоремы 1,2 из $[3$, гл. $7, \S 1]$ в случае нецелых $p_{+}$из $(0,2 n)$ и $p_{+}=2 n$, асимптотический метод, изложенный в $[5$, гл. $1, \S 3]$, при $p_{+}=1,2, \ldots, 2 n-1$ и, наконец, теорему 3 из $[3$, гл. $7, \S 1]$ в случае, когда $p_{+}>2 n$. Заметим, что проверка применимости перечисленных теорем и методов к нахождению асимптотики на бесконечности решений $\mathbf{v}(t)$ системы дифференциальных уравнений, к которой дифференциальное уравнения (3) приводится с помощью указанных выше преобразований, требует проведения достаточно громоздких вычислений.

ЗАмЕчАниЕ 2 . Пусть $y_{k}(x), 1 \leqslant k \leqslant n$, - первые $n$ функций, принадлежащих построенному в лемме 1 фундаментальному семейству решений дифференциального уравнения (3) с целым или нецелым значением параметра $p_{+} \in$ $(n-1 / 2,2 n)$. При $n+1 \leqslant j \leqslant 2 n$ первый член асимптотики в нуле производных $y_{k}^{(j-1)}$ указанных функций, получаемый при дифференцировании их асимптотики (5), становится неопределенным из-за того, что $\left(x^{k-1}\right)^{(j-1)}=0$ для рассматриваемых $k$ и $j$. Стандартный, но достаточно трудоемкий способ раскрытия этой неопределенности дает для любого $k, 1 \leqslant k \leqslant n$, оценки $y_{k}^{(j-1)}(x)=O\left(x^{2 n-p_{+}+k-j}\right), x \rightarrow+0$, при $n+1 \leqslant j \leqslant 2 n$, используемые в доказательстве теоремы 1.

Из асимптотики при $x \rightarrow+0$ функций, образующих фундаментальное семейство решений дифференциального уравнения (3), построенное в лемме 1 для $p_{+} \in(0,2 n)$ и лемме 2 для $p_{+} \geqslant 2 n$, легко определить, сколько из них принадлежит $L^{2}\left(I_{+}\right)$. Поэтому непосредственным следствием лемм 1,2 является

ЛЕмма 3. Индексы дефекта симметрического оператора $H\left(I_{+}\right)$, зависящего от параметра $p_{+}$, вычисляются по следующим формулам:

1) Def $H\left(I_{+}\right)=2 n$, если $p_{+}<n+1 / 2$;

2) $n+1 \leqslant \operatorname{Def} H\left(I_{+}\right) \leqslant 2 n-1 u$, более точно, Def $H\left(I_{+}\right)=3 n-\left[p_{+}+1 / 2\right]$, если $n+1 / 2 \leqslant p_{+}<2 n-1 / 2$

3) $\operatorname{Def} H\left(I_{+}\right)=n$, если $p_{+} \geqslant 2 n-1 / 2$.

ЗАмечАниЕ 3 . С учетом замечания 1 для оператора $H\left(I_{-}\right)$справедлив аналог леммы 2. Его формулировка получается путем замены в лемме 3 оператора $H\left(I_{+}\right)$и параметра $p_{+}$оператором $H\left(I_{-}\right)$и параметром $p_{-}$. 
Лемма 3 и замечание 3 позволяют получить теорему 2 с помощью следующих рассуждений.

Пусть $x_{r-1}, x_{r}$ - два соседних нуля коэффициента $c(x)$ дифференциального выражения $s[f]$, рассматриваемого в теореме 2 . Положим $J_{r}=\left(x_{r-1}, x_{r}\right)$ и покажем, что оператор $H\left(J_{r}\right)$, порожденный этим дифференциальным выражением, самосопряжен для любого $r,-\infty<r<+\infty$. В силу одного из условий теоремы 2 оба односторонних порядка каждого из нулей $x_{r-1}, x_{r}$ коэффициента $c(x)$ больше или равны $2 n-1 / 2$ и $c(x) \neq 0$ при $x \in J_{r}$. Зафиксируем какую-нибудь точку $\xi_{r} \in J_{r}$, после чего трем интервалам $J_{r}, J_{r,-}=\left(x_{r-1}, \xi_{r}\right), J_{r,+}=\left(\xi_{r}, x_{r}\right)$ поставим в соответствие минимальные операторы $H\left(J_{r}\right), H\left(J_{r,-}\right), H\left(J_{r,+}\right)$, порожденные рассматриваемым дифференциальным выражением $s[f]$ соответственно в $L^{2}\left(J_{r}\right), L^{2}\left(J_{r,-}\right), L^{2}\left(J_{r,+}\right)$. Их индексы дефекта связаны известной формулой (см., например, [4, гл. 13, §2, следствие 26])

$$
\operatorname{Def} H\left(J_{r}\right)=\operatorname{Def} H\left(J_{r,-}\right)+\operatorname{Def} H\left(J_{r,+}\right)-2 n \text {. }
$$

При сдвиге начала координат на оси переменной $x$ в точку $x_{r-1}$ (или в точку $x_{r}$ ) оператор $H\left(J_{r,-}\right)$ переходит в оператор $H\left(I_{+}\right)$, рассматриваемый в лемме 3 , с параметром $p_{+}=p_{+, r-1} \geqslant 2 n-1 / 2$ (соответственно оператор $H\left(J_{r,+}\right)$ переходит в оператор $H\left(I_{-}\right)$, рассматриваемый в замечании 3 , с параметром $p_{-}=$ $\left.p_{-, r} \geqslant 2 n-1 / 2\right)$. Поэтому из леммы 3 и замечания 3 следует, что $\operatorname{Def} H\left(J_{r,+}\right)=$ $\operatorname{Def} H\left(J_{r,-}\right)=n$. Подстановка полученных значений индексов дефекта операторов $H\left(J_{r,-}\right), H\left(J_{r,+}\right)$ в формулу $(6)$ дает равенство $\operatorname{Def} H\left(J_{r}\right)=0$, т. е. самосопряженность каждого из симметрических операторов $H\left(J_{r}\right),-\infty<r<+\infty$, действующих во взаимно ортогональных подпространствах $L^{2}\left(J_{r}\right)$ гильбертова пространства $L^{2}(\mathbb{R})$. Последнее свойство влечет за собой самосопряженность их бесконечной ортогональной суммы $\bigoplus_{r=-\infty}^{+\infty} H\left(J_{r}\right)$ в $L^{2}(\mathbb{R})$. Так как оператор $H$ является ее симметрическим расширением, то он совпадает с ней и, следовательно, самосопряжен. Таким образом, теорема 2 доказана.

\section{§3. Доказательство теоремы 1}

В отличие от случая теоремы 2, при доказательстве теоремы 1 недостаточно ограничиться сведениями об индексах дефекта симметрических операторов $H\left(I_{+}\right)$и $H\left(I_{-}\right)$, приведенными в леммах 1,2 . Нам также понадобятся определенные сведения об индексах дефекта действующего в $L^{2}\left(I_{0}\right), I_{0}=\left(-h_{-}, h_{+}\right)$, оператора $H\left(I_{0}\right)$, порожденного дифференциальным выражением $s[f]$ с коэффициентом $c(x)$, имеющим нуль $x=0$ двустороннего порядка $\left(p_{-}, p_{+}\right)$, т. е. допускающим представление (1). При получении этих сведений мы используем приведенное ниже в лемме 4 описание дефектного подпространства $\mathfrak{N}_{0} \subset L^{2}\left(I_{0}\right)$ оператора $H\left(I_{0}\right)$, отвечающего произвольному числу $\bar{z}, \operatorname{Im} z \neq 0$. В этом описании присутствуют отвечающие указанному комплексному числу дефектные подпространства $\mathfrak{N}_{+} \subset L^{2}\left(I_{+}\right), \mathfrak{N}_{-} \subset L^{2}\left(I_{-}\right)$минимальных операторов $H\left(I_{+}\right)$, $H\left(I_{-}\right)$, порожденных дифференциальными выражениями (2). В нем также используется билинейная форма $Q[f, y](x)$, определяющая вид внеинтегральных членов в тождестве Лагранжа $\int_{\alpha}^{\beta} s[f] \bar{y} d x=\int_{\alpha}^{\beta} f \overline{s[y]} d x+Q[f, y](\beta)-Q[f, y](\alpha)$; мы его рассматриваем для любого отрезка $[\alpha, \beta]$, принадлежащего одному из интервалов $I_{-}, I_{+}$, произвольной функции $f(x) \in C_{0}^{\infty}\left(I_{0}\right)$ и фиксированной функции $y(x)$, принадлежащей одному из классов $C^{\infty}\left(I_{-}\right), C^{\infty}\left(I_{+}\right)$. Нам понадобятся обозначения $Q[f, y]( \pm 0)=\lim _{x \rightarrow \pm 0} Q[f, y](x)$. 
С помощью простых вычислений можно получить представление

$$
Q[f, y](x)=Q_{1}[f, y](x)+Q_{2}[f, y](x),
$$

где

$$
Q_{1}[f, y](x)=\sum_{j=1}^{n} q_{j}[f](x) \overline{y^{(j-1)}(x)}, \quad Q_{2}[f, y](x)=\sum_{j=n+1}^{2 n} q_{j}[f](x) \overline{y^{(j-1)}(x)}
$$

здесь для любого $j, 1 \leqslant j \leqslant 2 n$, через $q_{j}[f](x)$ обозначено линейное дифференциальное выражение от $f(x)$ порядка $2 n-j$ с коэффициентами, выражающимися через производные тех или иных порядков от коэффициента $c(x)$ дифференциального выражения $s[f]$.

Лемма 4. Дефектное подпространство $\mathfrak{N}_{0}$ оператора $H\left(I_{0}\right)$ coвпадает с совокупностью функиий $y(x) \in L^{2}\left(I_{0}\right)$, обладающих следующими свойствами:

1) $y(x) \in \mathfrak{N}_{-} \oplus \mathfrak{N}_{+}$;

2) односторонние предель $Q[f, y](-0)$ и $Q[f, y](+0)$ существуют для любой функиии $f(x) \in C_{0}^{\infty}\left(I_{0}\right)$;

3) для указанных $f(x)$ выполняется условие сопряжения $Q[f, y](-0)=$ $Q[f, y](+0)$.

Доказательство леммы 4 имеет элементарный характер.

Доказательство теоремы 1 опирается на следующее свойство индексов дефекта оператора $H\left(I_{0}\right)$, зависящего от параметров $p_{-}, p_{+}$.

ЛЕмма 5. Пусть двусторонний порядок $\left(p_{-}, p_{+}\right)$нуля $x=0$ коэфбициента $c(x)$ дифференщиального выражения $s[f]$, порождающего действующий $в$ $L^{2}\left(I_{0}\right)$ симметрический минимальный оператор $H\left(I_{0}\right)$, удовлетворяет условию $n-1 / 2<p_{-}, p_{+}<2 n-1 / 2$. Тогда справедливо неравенство Def $H\left(I_{0}\right) \geqslant$ $2 n+1$.

Для доказательства леммы 5 достаточно при ограничениях, наложенных в ней на $p_{-}, p_{+}$, построить $2 n+1$ линейно независимых функций $y(x), x \in$ $\left(-h_{-}, h_{+}\right)$, принадлежащих дефектному подпространству $\mathfrak{N}_{0}$ оператора $H\left(I_{0}\right)$, отвечающему какому-нибудь числу $\bar{z}, \operatorname{Im} z \neq 0$, т. е. обладающих тремя свойствами, перечисленными в лемме 4.

Первые два из этих свойств относятся к ограничениям функций $y(x)$ на интервалы $\left(-h_{-}, 0\right),\left(0, h_{+}\right)$и симметрическим минимальным операторам $H\left(I_{-}\right)$ и $H\left(I_{+}\right)$, порожденным дифференциальным выражением $s[f]$ из леммы 5 соответственно в гильбертовых пространствах $L^{2}\left(I_{-}\right)$и $L^{2}\left(I_{+}\right)$. В силу замечания 1 указанные свойства достаточно изучать только для ограничений функций $y(x)$ на интервал $\left(0, h_{+}\right)$.

Будем считать, что параметр $p_{+} \in(n-1 / 2,2 n-1 / 2)$ принимает нецелые значения. В силу первого утверждения леммы 1 при сделанном предположении для получения базиса в рассматриваемом в лемме 4 дефектном подпространстве $\mathfrak{N}_{+}$оператора $H\left(I_{+}\right)$достаточно выбрать из фундаментального семейства решений $y_{k}(x), 1 \leqslant k \leqslant 2 n$, дифференциального уравнения (3), асимптотика которых при $x \rightarrow+0$ приведена в формуле (5), решения, принадлежащие $L^{2}\left(I_{+}\right)$. Легко проверяется, что базис в $\mathfrak{N}_{+}$составляют, во-первых, $n$ решений $y_{k}(x)$, $1 \leqslant k \leqslant n$, и, во-вторых, $2 n-\left[p_{+}+1 / 2\right] \geqslant 1$ решений $y_{k}(x),[p+1 / 2]+1 \leqslant k \leqslant 2 n$. В частности, для любого нецелого $p_{+} \in(n-1 / 2,2 n-1 / 2)$ указанному базису в $\mathfrak{N}_{+}$принадлежит функция $y_{2 n}(x)$. Следующим шагом в доказательстве леммы 5 является такое вспомогательное утверждение. 
ЛЕмма 6. При $1 \leqslant k \leqslant n$ u $k=2 n$ для любой функиии $f(x) \in C_{0}^{\infty}\left(I_{0}\right)$ существуют односторонние предель $Q\left[f, y_{k}\right](+0)=\lim _{x \rightarrow+0} Q\left[f, y_{k}\right](+x)$, причем справедливы равенства

$$
Q\left[f, y_{k}\right](+0)=0, \quad 1 \leqslant k \leqslant n, \quad Q\left[f, y_{2 n}\right](+0)=C\left(p_{+}\right) a_{+}(0) f(0)
$$

константа $C\left(p_{+}\right)$является значением при $p=p_{+}$некоторой функции $C(p)$ параметра $p$, обладающей свойством $C(p) \neq 0$ для нецелых $p$.

Для проверки справедливости леммы 6 следует вычислять указанные в ней пределы, заменяя присутствующие в формулах $(7),(8)$ функции $c(x)=x^{p_{+}} a_{+}(x)$, $f(x) \in C_{0}^{\infty}\left(I_{0}\right)$, а также решения $y_{k}(x), 1 \leqslant k \leqslant n$ и $k=2 n$, дифференциального уравнения (3) и их производные асимптотиками перечисленных функций при $x \rightarrow+0$. Пусть $s^{\{0\}}=1, s^{\{m\}}=\prod_{l=0}^{m-1}(s-l), m=1,2, \ldots$, - обобщенные степени числа $s$. Если воспользоваться асимптотиками функции $y_{2 n}(x)$ и ее производных до порядка $2 n-1$, вытекающими из формулы (5), то на этом пути тривиально получается второе равенство формулы (9) с константой $C\left(p_{+}\right)$- значением при $p=p_{+}$функции $C(p)=(-1)^{n-1} \sum_{j=n+1}^{2 n} C_{n-1}^{j-1-n} p^{\{2 n-j\}}(2 n-1-p)^{\{j-1\}}$. $\mathrm{C}$ помощью некоторых формул теории конечных разностей устанавливается, что $C(p)=(-1)^{n-1}(n-1) !(2 n-1-p)^{\{n\}}$, и, следовательно, $C(p) \neq 0$ для нецелых значений $p$. При вычислении (с использованием тех же технических средств) пределов $Q\left[f, y_{k}\right](+0), 1 \leqslant k \leqslant n$, выясняется, что для первой из сумм (8) легко выводится равенство $\lim _{x \rightarrow+0} Q_{1}\left[f, y_{k}\right](x)=0$, однако при вычислении аналогичного предела второй из сумм (8) возникает неопределенность, связанная с обстоятельством, отмеченным в замечании 2. Эта неопределенность раскрывается с помощью приведенной в этом замечании асимптотической оценки производных порядков $j-1, n+1 \leqslant j \leqslant 2 n$, от функций $y_{k}(x), 1 \leqslant k \leqslant n$. Такая оценка достаточна для обоснования равенства $\lim _{x \rightarrow+0} Q_{2}\left[f, y_{k}\right](x)=0$, из которого следуют в силу формул (7), (8) первые $n$ из равенств (9), т. е. справедливость леммы 6.

Согласно замечанию 1 , из установленных выше фактов, относящихся к оператору $H\left(I_{+}\right)$, зависящему от нецелого параметра $p_{+} \in(n-1 / 2,2 n-1 / 2)$, вытекают аналогичные факты для оператора $H\left(I_{-}\right)$. Поэтому для нецелых $p_{-} \in$ $(n-1 / 2,2 n-1 / 2)$ в дефектном подпространстве $\mathfrak{N}_{-}$оператора $H\left(I_{-}\right)$, рассматриваемом в лемме 4 , существует базис, из которого можно выбрать $n+1$ функций (обозначим их через $\left.\eta_{k}(x), 1 \leqslant k \leqslant n, \eta_{2 n}(x)\right)$, для которых справедлив аналог леммы 6 . Это означает, что $Q\left[f, \eta_{k}\right](-0)=0,1 \leqslant k \leqslant n, Q\left[f, \eta_{2 n}\right](-0)=$ $C\left(p_{-}\right) a_{-}(0) f(0), C\left(p_{-}\right) \neq 0$. Используя функции $\eta_{k}(x), 1 \leqslant k \leqslant n, \eta_{2 n}(x)$ вместе с функциями $y_{k}(x), 1 \leqslant k \leqslant n, y_{2 n}(x)$, рассматриваемыми в лемме 6 , построим $2 n+1$ функций $u_{k}(x) \in L^{2}\left(I_{0}\right),-n \leqslant k \leqslant n$, первые $n$ из которых (отвечающие индексам $-n \leqslant k \leqslant-1)$ равны $\eta_{-k}(x)$ при $x \in I_{-}$и нулю при $I_{+}$, последние $n$ (отвечающие индексам $1 \leqslant k \leqslant n$ ) равны функциям $y_{k}(x)$ при $x \in I_{+}$и нулю при $x \in I_{-}$и, наконец, функция $u_{0}(x)$ имеет вид $C\left(p_{+}\right) a_{+}(0) \eta_{2 n}(x)$ на интервале $I_{-}$и $C\left(p_{-}\right) a_{-}(0) y_{2 n}(x)$ на интервале $I_{+}$. Эти функции линейно независимы на интервале $I_{0}$ и обладают свойствами, перечисленными в лемме 4 . По лемме 4 они принадлежат дефектному подпространству $\mathfrak{N}_{0}$ оператора $H\left(I_{0}\right)$, и, следовательно, размерность Def $H\left(I_{0}\right)$ последнего больше или равна $2 n+1$. Так устанавливается справедливость леммы 5 для нецелых значений параметров $p_{-}, p_{+}$, удовлетворяющих ее условию. 
Пусть теперь параметр $p_{+} \in(n-1 / 2,2 n-1 / 2)$ принимает одно из целых значений $n, \ldots, 2 n-1$. Рассмотрим соответствующее фундаментальное семейство решений $y_{k}(x), 1 \leqslant k \leqslant 2 n$, дифференциального уравнения (3), описание $2 n-1$ раз дифференцируемой асимптотики которых при $x \rightarrow+0$ приведено во втором утверждении леммы 1. С помощью вычислений, которые в рассматриваемом случае приходится выполнять заново, проверяется, что для функций $y_{k}(x)$, $1 \leqslant k \leqslant n$, и $y_{2 n}(x)$ остается в силе лемма 6 . Таким образом, фундаментальное семейство решений $y_{k}(x), 1 \leqslant k \leqslant 2 n$, дифференциального уравнения (3), обладающее свойствами (9), существует для любых $p_{+} \in(n-1 / 2,2 n-1 / 2)$. Поэтому заключительная часть доказательства леммы 5 дает обоснование этой леммы не только для нецелых $p_{-}, p_{+}$, но и для любых значений этих параметров, удовлетворяющих ее условию. Таким образом, лемма 5 справедлива в полной общности.

ЗАмЕчАНИЕ 4. Заменим присутствующее в лемме 5 двустороннее ограничение на параметры $p_{-}, p_{+}$менее ограничительным односторонним вида $p_{-}, p_{+}>$ $n-1 / 2$. Простой анализ доказательства этой леммы (с привлечением не только леммы 1 , но и леммы 2) показывает, что в последнем случае оценка $\operatorname{Def} H\left(I_{0}\right) \geqslant$ $2 n+1$ может не быть справедливой, однако обязательно выполняется более слабое неравенство Def $H\left(I_{0}\right) \geqslant 2 n$.

В доказательстве теоремы 1 , основанном на лемме 5 , будем использовать обозначения $\mathbb{R}_{x}^{+}=(x,+\infty), \mathbb{R}_{x}^{-}=(-\infty, x)$ и называть ячейкой произвольный ограниченный интервал $I=(\alpha, \beta)$, содержащий в точности один нуль коэффициента $c(x)$ дифференциального выражения $s[f]$, удовлетворяющего условиям этой теоремы, и такой, что $c(\alpha) c(\beta) \neq 0$. Пусть $H$ - симметрический минимальный оператор в $L^{2}(\mathbb{R})$, порожденный дифференциальным выражением $s[f]$.

Зафиксируем точку $\xi_{0}$, обладающую свойством $c\left(\xi_{0}\right) \neq 0$, которая расщепляет числовую ось на полуоси $\mathbb{R}_{\xi_{0}}^{-}$и $\mathbb{R}_{\xi_{0}}^{+}$. По крайней мере одна из этих полуосей содержит бесконечно много нулей коэффициента $c(x)$, двусторонние порядки $p_{-}, p_{+}$которых удовлетворяют условию теоремы 1 ; для определенности, предположим, что таким свойством обладает полуось $\mathbb{R}_{\xi_{0}}^{+}$. Нули коэффициента $c(x)$, принадлежащие полуоси $\mathbb{R}_{\xi_{0}}^{+}$, образуют возрастающую последовательность $\left\{x_{r}\right\}_{r=1}^{\infty}, \lim _{r \rightarrow+\infty} x_{r}=+\infty$. В каждом из интервалов $\left(x_{r}, x_{r+1}\right)$ выберем по точке $\xi_{r}$ и рассмотрим последовательность ячеек $I_{r}=\left(\xi_{r-1}, \xi_{r}\right), 1 \leqslant r<\infty$, содержащих по одному нулю $x_{r}$ коэффициента $c(x)$. Применяя формулу $(6) \mathrm{k}$ полуоси $J_{r}=\mathbb{R}_{\xi_{r-1}}^{+}$, расщепленной точкой $\xi_{r}$ на интервал $J_{r,-}=I_{r}$ и полуось $J_{r,+}=\mathbb{R}_{\xi_{r}}^{+}$, при каждом $r$ и суммируя первые $N$ таких равенств, получим равенство Def $H\left(\mathbb{R}_{\xi_{0}}^{+}\right)=S_{N}+\operatorname{Def} H\left(\mathbb{R}_{\xi_{N+}^{+}}^{+}\right)$, где $S_{N}=\sum_{r=1}^{N}\left(\operatorname{Def} H\left(I_{r}\right)-2 n\right)$. Следствием этой формулы является оценка Def $H=\operatorname{Def} H\left(\mathbb{R}_{\xi_{0}}^{-}\right)+\operatorname{Def} H\left(\mathbb{R}_{\xi_{0}}^{+}\right)-2 n \geqslant$ $S_{N}-2 n$ для любого сколь угодно большого $N$.

Согласно нашему предположению, существует подпоследовательность ячеек $I_{r_{l}}, 1 \leqslant l<\infty$, с нулями $x_{r_{l}}$ коэффициента $c(x)$, двусторонние порядки $\left(p_{-}, p_{+}\right)$ которых удовлетворяют условию теоремы 1 . При сдвиге точки $x=0$ в каждый из нулей $x_{r_{l}}$ оператор $H\left(I_{r_{l}}\right)$ преобразуется в оператор $H\left(I_{0}\right)$, порожденный дифференциальным выражением $s[f]$ с коэффициентом, удовлетворяющим условиям леммы 5. По этой лемме Def $H\left(I_{r_{l}}\right)=\operatorname{Def} H\left(I_{0}\right) \geqslant 2 n+1,1 \leqslant l<\infty$. 
Аналогично с помощью замечания 4 показывается, что Def $H\left(I_{r}\right) \geqslant 2 n$ для ячеек $I_{r}$, не принадлежащих указанной подпоследовательности. Так как из последних неравенств следует, что сумма $S_{N}$ неограниченно возрастает при $N \rightarrow \infty$, то Def $H=+\infty$. Теорема 1 доказана.

\section{§4. Заключительные замечания}

Мы сформулировали теоремы 1 и 2 в их простейшем варианте - при условии бесконечной дифференцируемости коэффициента $c(x)$ в точках $x$, в которых $c(x) \neq 0$, и в предположении отсутствия конечных предельных точек у множества его нулей. Для применимости используемых в доказательствах этих теорем асимптотических методов достаточно конечной гладкости функции $c(x)$ в указанных точках, однако ее порядок неограниченно возрастает при $n \rightarrow+\infty$. Простой анализ доказательств теорем 1, 2 показывает, что их утверждения остаются справедливыми и тогда, когда множество нулей этой функции имеет ограниченное количество конечных предельных точек.

Пусть нули коэффициента $c(x)$ дифференциального выражения $s[f]$ порядка $2 n \geqslant 2$ образуют возрастающую последовательность $x_{r},-\infty<r<+\infty$, $\lim _{r \rightarrow \pm \infty} x_{r}= \pm \infty$, удовлетворяющую условиям теоремы 2. Нетрудно проверить, что рассуждения, с помощью которых доказывается эта теорема, применимы не только к соответствующему оператору $H$, но и к более общему симметрическому минимальному оператору $H_{q}$ в $L^{2}(\mathbb{R})$, порожденному дифференциальным выражением $s_{q}[f]=s[f]+q(x) f$ с любым действительным потенциалом $q(x) \in L_{\mathrm{loc}}^{\infty}(\mathbb{R})$, независимо от его поведения на бесконечности. Это означает, что теорема 2 верна также для оператора $H_{q}$. Аналогично, если порядки нулей рассматриваемого коэффициента $c(x)$ удовлетворяют условиям теоремы 1 , для оператора $H_{q}$ остается в силе утверждение этой теоремы. Такие обобщения теорем 1,2 , в которых отсутствуют ограничения на поведение потенциала $q(x)$ на бесконечности, не имеют аналогов в случае, когда коэффициент $c(x)$ дифференциального выражения $s_{q}[f]$ строго положителен на числовой оси.

Интересно отметить, что утверждение теоремы 1 о бесконечности индексов дефекта симметрического минимального оператора $H$ в $L^{2}(\mathbb{R})$, порожденного дифференциальным выражением $s[f]=(-1)^{n}\left(c(x) f^{(n)}\right)^{(n)}, n \geqslant 1$, с коэффициентом $c(x)$, удовлетворяющим ее условию, равносильно существованию для любого числа $z, \operatorname{Im} z \neq 0$, бесконечного множества линейно независимых решений $y(x) \in L^{2}(\mathbb{R})$ дифференциального уравнения $(-1)^{n}\left(c(x) y^{(n)}\right)^{(n)}=z y$ в смысле обобщенных функций.

\section{ЛитерАтУРА}

1. Орочко Ю. Б. Функц. анализ и его прил., 28, 69-72 (1994).

2. Хартман Ф. Обыкновенные дифференциальные уравнения. Мир, М., 1970.

3. Наймарк M. А. Линейные дифференциальные операторы. Наука, М., 1969.

4. Данфорд Н., Швари, Дж. Т. Линейные операторы. Спектральная теория. Самосопряженные операторы в гильбертовом пространстве. Мир, М., 1966.

5. Panonopm И. М. О некоторых асимптотических методах в теории дифференциальных уравнений. Изд-во АН УССР, Киев, 1954. 